\title{
From Cognitive Science to Physics Education and Back
}

\author{
Sian L. Beilock* and Susan M. Fischer† \\ *Department of Psychology, The University of Chicago, 5848 South University Ave. Chicago, IL 60637 \\ $\dagger$ Department of Physics, DePaul University, 2219 North Kenmore Avenue, Chicago, IL 60614
}

\begin{abstract}
Principles of learning and performance derived from research in cognitive science can inform how physics is taught and how learning is assessed. At the same time, common practices in physics education can be used to develop better cognitive principles of student learning and understanding. This paper has two main themes. First, we discuss how we can use psychology and neuroscience research regarding how academic anxiety alters thinking and reasoning to help students perform at their best - especially on important tests. Second, we explore how basic principles of learning can be used to develop optimal labs in physics education settings. Finally, we end by discussing how, together, PER and cognitive science can be used to help students perform at their best when it matters most.
\end{abstract}

Keywords: Learning, Stress, Math Anxiety, Emotion, Motivation, Embodied Cognition

PACS: 01.40.-d

\section{INTRODUCTION}

It is easy to think that our only goal as teachers and educators is to provide students with knowledge and skill. However, student success is based on more than just what they know in a particular subject area. Students' attitudes, anxieties and motivations are also key.

In this paper, we take a two pronged approach to thinking about success in physics. First, we discuss some of the psychological impediments to physics achievement - particularly how stressful academic situations can negatively impact student learning and performance. We argue that by understanding what happens in the brain and body in situations where students feel pressure to perform well, we can develop pretty simple psychological tools - that neither require a lot of time, money nor investment - to help students perform at their best when it matters most. Second, we also explore how basic principles of learning can be used to develop optimal labs in physics education settings. Together, this work illustrates how cognitive science can be used to inform Physics Education Research (PER) and vice versa.

\section{ACADEMIC PERFORMANCE AND STRESS: THE CASE OF MATH ANXIETY}

It is no secret that math skills are important for success in the physics classroom. Yet many students experience apprehension and fear when dealing with numerical information, termed math anxiety. Math anxiety is an adverse emotional reaction to math or the prospect of doing math and goes hand-in-hand with low achievement in math and subjects that require math (e.g., physics). [1]
The relation between math anxiety and poor math performance starts early in schooling. For students as young as first grade, the higher their math anxiety, the lower their math achievement at the end of the school year. [2] This same relation is seen all the way through the college years and into adulthood.

Where does math anxiety come from? One source of negativity surrounding math, at least early on in schooling, is students' elementary school teachers. As it happens, teachers who are anxious about their own math abilities impart these negative attitudes to some of their students. Interestingly, this transmission of negative math attitudes seems to fall along gender lines. Beilock and colleagues found that female students of highly math anxious female $1^{\text {st }}$ and $2^{\text {nd }}$ grade teachers $(>90 \%$ of elementary teachers in the USA are female) tended to endorse the stereotype that 'boys are good at math, girls at reading' by the end of a school year. [3] Girls who endorsed this stereotype were also most likely to be behind in math at the end of the school year. Similar results have been found in college physics classes. Women who tend to endorse the stereotype that men do better than women in physics perform more poorly than their classmates. [4]

Turning back to math anxiety, why is math anxiety tied to poor math performance? One idea is that math anxiety is simply a proxy for low math ability, meaning that students with math anxiety are less skilled at math than their non-anxious counterparts. Yet low math ability does not seem to be the entire explanation for why math anxiety and poor math performance co-occur. In our lab, we have shown that people's anxiety about doing math - over and above their actual math ability - is an impediment to math achievement. 
When faced with a math task, math anxious individuals tend to worry about the situation and its consequences. These worries compromise cognitive resources, such as working memory, a short-term system involved in the regulation and control of information relevant to the task at hand. [5] When the ability of working memory to maintain task focus is disrupted, academic performance can suffer.

It is also the case that, in adults, just the knowledge that one will have to later perform a math task can send math anxious individuals into a panic. Lyons and Beilock $^{6}$ recently showed that when simply anticipating an upcoming math task, the higher one's math anxiety, the more one increases activity in brain regions associated with visceral threat detection and often the experience of pain itself (see Figure 1). [6] Our data suggest that activation of neural regions implicated in physical pain underlies the intuition that simply anticipating a dreaded event can feel painful.

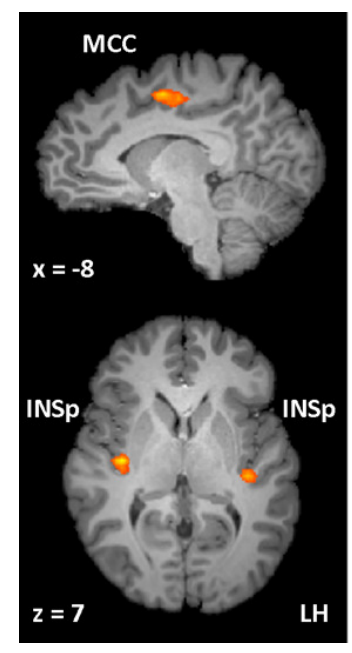

FIGURE 1. Brain regions associated with math anxious individuals' anticipation of doing math. For full explanation, see Lyons \& Beilock (2011). [6] INSp: dorso-posterior insula; MCC: mid-cingulate cortex; LH: Left hemisphere

Understanding math anxiety (e.g., when it comes into play in formal schooling, and how it relates to performance in math and science throughout schooling) is critical for devising ways to alleviate its negative impact and the negative impact of stressful performance situations on test performance in math and science more broadly.

One means by which people can regulate their negative emotions is expressive writing in which people are asked to write freely about their emotions for 10-15 min with respect to a specific situation (e.g. an upcoming physics exam). Writing is believed to alleviate the burden that negative thoughts place on working memory by affording people an opportunity to re-evaluate the stressful experience in a manner that reduces the necessity to worry altogether.

Illustrating the benefits of expressive writing, Ramirez and Beilock showed that having highly test anxious high school students write about their worries prior to an upcoming biology final exam boosted their scores from a B- to $\mathrm{B}+$ on average (even after taking into account grades across the school year). [7] We have recently found similar effects specifically for math anxiety. Writing about math-related worries boosts the math test scores of math anxious students.

It is also the case that giving students the opportunity to take many practice tests can help lessen anxiety on the big testing day. Studying under the same conditions you will be tested under - for instance, in a timed situation with no study aids - helps you get used to what you will experience on test day. There is also research suggesting that testing yourself on material (rather than simply studying it) helps you remember it better in the long term - this is termed "the testing effect". [8] If students are going to be tested during an exam, practice being tested can help.

In sum, how a student performs in the classroom is more than about what they know - their anxieties and attitudes are also critical. In physics, where mathematics is a mainstay of success in many classes, addressing the anxiety that is associated with math itself may be an important way to enhance engagement, motivation, and performance. But, there is more. Understanding something about how mathematics anxiety works (i.e., the cognitive and neural differences between low and high math anxious students) can also inform how we think about learning specific concepts in the physics classroom.

In particular, refer back to Figure 1. This figure illustrates that when anticipating an upcoming mathtask, the higher one's math anxiety, the more one increases activity in regions associated with bodily threat detection and the experience of visceral pain itself (e.g., the posterior portion of a brain region known as the Insula). The finding that anticipatory anxiety about math is grounded in the simulation of visceral threat and even pain suggests that our neural hardware doesn't always make a distinction between what is real and what isn't (i.e., actual pain versus the anticipation of a psychologically painful event). Our research group has been capitalizing on this fuzzy line between the mental and physical to explore how best to advance learning in the physics classroom. [9] We call it embodied physics learning.

\section{EMBODIED PHYSICS LEARNING}

The central tenet of our embodied physics learning work is that providing students with direct experience with physics quantities (e.g., feeling forces - as 
opposed to reading about force, seeing forces being exerted on someone else, or even measuring forces with instruments) can enhance student understanding. Lab experiences where students feel physics quantities may lead to the recruitment of brain areas devoted to sensory and motor (sensorimotor) processing when students later think and reason about the physics concepts they experienced. When these sensorimotor areas are involved in thinking and reasoning tasks, people's understanding of the concepts in question can improve. [10-13]

Just like math anxious individuals activate brain areas implicated in pain perception when they are thinking about doing math, our idea is that if we can engage students' sensorimotor brain systems when they simply think about a physics concept that they have experienced previously, they may be better equipped to understand and apply that concept even when they are not acting. Moreover, experiences that engage the sensorimotor system may be especially important at the initial stages of learning when students are first being formally introduced to a concept. Initial sensorimotor experiences may also help students benefit from virtual labs presented later in learning by providing added meaning to learning tools such as computer simulations.

We have performed several experiments that represent an initial exploration of whether labs where students become an active part of a physical system can be used to increase student proficiency. The first of these focuses on student understanding of the concept of angular momentum.

The angular momentum of a spinning object is defined as the product of the moment of inertia of the object and the angular velocity of the object. The moment of inertia of an object depends on its mass and on the way the mass is distributed. The farther the mass is from the axis of rotation of the object, the larger the moment of inertia. The angular velocity corresponds to the speed of rotation, and might be given in revolutions/second.

Our study used a system of two bicycle wheels that spun independently about a single axle (see Figure 2) to demonstrate these concepts. In this system, a set of larger radius wheels will have a larger moment of inertia than a set of smaller radius wheels. The faster a wheel spins, the larger its angular velocity and angular momentum. When the wheels do not spin at all, the angular momentum is zero. But angular momentum has the additional feature of being a vector quantity with an associated direction. For our bicycle wheels, the angular momentum vector always points along the axle of the wheels. It is the consequences of this vector nature of angular momentum that one feels directly by handling the spinning wheels and tilting the axle. In our studies, students systematically varied the wheel size, tilt speed of the axle, spin speed, and spin direction as they repeatedly changed the orientation of the wheels from vertical, to horizontal, and back to vertical.

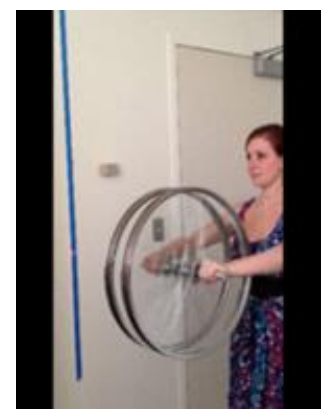

FIGURE 2. Spinning bicycle wheel demonstration

We have demonstrated the benefits of sensorimotor experience for students' understanding of the angular momentum concept across several experiments in the psychology laboratory, physics classroom, and using a neuroimaging technique called fMRI (functional Magnetic Resonance Imaging). We refer to the ability of sensorimotor experience to modify sensorimotor representations - and for students to call upon these modified representations even when they are not acting (in this case, handling the wheels) - as embodied learning.

Specifically, our work has shown that students who became part of the physical system and felt the effects of a changing angular momentum themselves (i.e., sensorimotor or action experience) developed better understanding of factors influencing angular momentum than students who only observed a demonstration of angular momentum and torque (i.e., observation experience). Importantly, visual input and attentional demands were always equated across our learning experiences, suggesting that sensorimotor experience drove learning differences.

We have shown a positive impact of our sensorimotor experience on student understanding of the factors which influence angular momentum and torque in both the psychology laboratory (where subjects are not students in a physics course) and in authentic physics classroom settings on traditional quiz and homework assignments. Moreover, using fMRI to look at neural activation of students who had motor experience versus those that did not when they were later solving quiz questions on the topic of angular momentum, we found that the degree of involvement of sensorimotor brain systems relates to understanding. In particular, we found that sensorimotor training (relative to observation) leads to increased activation of sensorimotor systems important for representing dynamic physical concepts when just thinking about 
the concepts in question. This activation, in turn, enhances understanding of torque and angular momentum.

In sum, our goal is to develop classroom exercises and activities along with a set of guiding learning principles built out of cognitive science theories of embodied cognition that allow students to attach a physical meaning to concepts and problem-solving techniques. Although we have much more work to do, we hope that our research group's understanding of psychology and neuroscience, coupled with expertise in PER, will ultimately lead to the development of optimal learning experiences in the physics classroom.

\section{CONCLUSION}

In conclusion, in this paper we discuss how we can use psychology and neuroscience research regarding how academic anxiety alters thinking and reasoning to enhance student learning and performance in the classroom. Second, we explore how basic principles of learning can be used to develop optimal labs in physics education settings. We aim to use cognitive science principles around learning and emotion to advance student achievement by infusing cognitive science into PER, and using PER to better understand how the mind works.

\section{ACKNOWLEDGMENTS}

This work is supported by NSF CAREER grant DRL0746970 and IES grant R305A110682 to S.L.B., and by NSF FIRE DRL-1042955 and a Spencer Foundation Grant 201000085 to S.L.B and S.F. This work is also supported by the NSF Spatial Intelligence and Learning Center.

\section{REFERENCES}

1. Hembree, R. (1990). J. Res. Math. Educ. 21, 33-46

2. Ramirez, G. et al. (2012). J. Cogn. Dev. http://dx.doi.org/ 10.1080/15248372.2012.664593

3. Beilock, S.L. et al. (2010). Proc. Natl. Acad. Sci. U.S.A. 107, 1060-1063

4. Miyake, A. et al. (2010). Science, 330, 12344-1237.

5. Beilock, S.L. (2010) Choke: What the Secrets of the Brain Reveal about Getting It Right When You Have To, Simon \& Schuster

6. Lyons, I. M., \& Beilock, S. L. (2012). PLOS ONE, 7, e48076.

7. Ramirez, G. and Beilock, S.L. (2011). Science 331, 211-213.

8. Roediger, H. L., \& Karpicke, J. D. (2006). Perspectives on Psychological Science, 1: 181-210.

9. Kontra, Lyons, Fischer \& Beilock. Manuscript in preparation.
10. Beilock, S. L. et al. (2008). Proc. Natl. Acad. Sci. U.S.A., 105, 13269-13273.

11. Barsalou, L. W. et al. (2003). Trends in cognitive sciences, 7(2), 84-91.

12. Kontra, C. E., Goldin-Meadow, S., \& Beilock, S. L. (2012). Topics in Cognitive Science, 1-9.

13. Glenberg, A. M. et al. (2004). J. Ed. Psych., 96(3), 424. 